\title{
Peripheral nerve block for anesthesia in patients having knee arthroplasty
}

\author{
Young-Tae Jeon \\ Department of Anesthesiology and Pain Medicine, Seoul National University Bundang Hospital, Seongnam, Korea
}

Total knee arthroplasty is an effective surgical modality for osteoarthritis of the knee. Because the population is aging, the number of TKA procedures is increasing every year [1]. Both general and diverse types of regional anesthesia have been used successfully for this surgery. Femoral nerve block (FNB) was first described in 1973 and used either as a single nerve or 3-in-1 block (including the femoral nerve, the lateral cutaneous nerve, and the obturator nerve), both as an independent anesthetic modality and as an analgesic adjunct to other anesthetic techniques.

In this issue of Korean Journal of Anesthesiology, Kim et al. [2] reported a study of peripheral nerve block for total knee arthroplasty. The authors conclude that femoral/sciatic nerve block with lateral femoral cutaneous nerve block can be an alternative to spinal epidural anesthesia in patients having total knee arthroplasty. They did not recommend peripheral nerve blockade as a first choice, because it is associated with a higher use of antihypertensives, analgesics, and sedatives. Nonetheless, peripheral nerve blockade showed comparable patient satisfaction and postoperative analgesia to spinal epidural anesthesia.

This report is likely to create controversy because a higher use of antihypertensives, analgesics, and sedatives are associated with incomplete anesthesia. FNB does not consistently produce anesthesia of the obturator nerve [3]. The obturator nerve or sciatic nerve supplies the knee joint. Consequently, the sensation in the back of the knee can be intact after FNB. Sciatic innervations in the back of the knee is a relatively minor contribution to postoperative pain after TKA [4].
The authors argued that sparing the obturator nerve is the main cause of insufficient anesthesia. In the previous report, the addition of the obturator nerve block to FNB improves postoperative analgesia after TKA [5]. In contrast, Bouaziz et al.[6] showed that efficacy of the obturator nerve block is not sufficient as the cutaneous contribution of the obturator nerve is absent in $57 \%$ of patients. Further investigations are required to confirm the anesthetic effect of obturator nerve block for TKA.

Recently, peripheral nerve blocks are widely used for low extremity surgery, but they are still less common spinal anesthesia, mainly because of the increased time and expertise required as well as the delayed recovery associated with their use. The increased time required to perform peripheral nerve block can be reduced by placing peripheral nerve blocks preoperatively in the block room.

Compared with neuroaxial (spinal/epidural) anesthesia, FNB minimizes hypotension, urinary retention, pruritis, and eliminates the risk of spinal hematoma and infection. Moreover, patients with hemodynamical instability, antiplatelet, or anticoagulant therapy can undergo a lower extremity nerve block without significant risk. The anatomical deformity caused by a degenerative change in elderly patients occasionally makes neuroaxial block inappropriate to surgical anesthesia for low extremity surgery. These benefits of lower extremity peripheral nerve block satisfy its use as an alternative anesthesia in patients undergoing lower extremity surgery. Despite these benefits, complications of peripheral nerve block limit its use for surgery. The common complications of FNB include

Corresponding author: Young-Tae Jeon, M.D., Ph.D., Department of Anesthesiology and Pain Medicine, Seoul National University Bundang Hospital, 300, Gumi-dong, Bundang-gu, Seongnam 463-802, Korea. Tel: 82-31-787-7493, Fax: 82-31-787-4063, E-mail: ytjeon@snubh.org (c) This is an open-access article distributed under the terms of the Creative Commons Attribution Non-Commercial License (http:// creativecommons.org/licenses/by-nc/3.0/), which permits unrestricted non-commercial use, distribution, and reproduction in any medium, provided the original work is properly cited. 
incomplete nerve blockade, direct nerve trauma with potential quadriceps wasting, local hematoma and subsequent ischemic injury, infection, and the risks of systemic intravenous injection of the local anesthetic agent.

A single injection of local anesthetic in FNB results in quadriceps muscle weakness and inability to ambulate for most of the duration of local anesthetics. This is important because there is an increasing trend to decrease time to early ambulation, even on the same day of surgery. Although a large study showed that long-acting peripheral nerve block of the lower extremity may be performed safely and is associated with a high degree of efficacy and satisfaction [7], a serious complication occurred in 4 patients, each of whom fell and sustained further injury after the use of femoral nerve blockade [8]. After the falls, neurological examination of the operated legs revealed reduced 2-point discrimination, pain and light touch sensation. An additional 4 falls were reported among 233 patients who received a femoral perineural catheter for pain management at home [9]. These findings suggest that this practice may not be free of risk. After having a femoral nerve block, patients should undergo enhanced postoperative evaluation of blockade and prorioceptive function to ensure safe neurological function before mobilization.

At this point in time, FSNB is not a good alternative to spinalepidural anesthesia; it is a final option when spinal-epidural anesthesia is not available. If FOS (femoral, obturator, sciatic) NB provides sufficient anesthesia for TKA, peripheral nerve block can be a good anesthetic candidate for TKA.

\section{References}

1. Kurtz S, Ong K, Lau E, Mowat F, Halpern M. Projections of primary and revision hip and knee arthroplasty in the United States from 2005 to 2030. J Bone Joint Surg Am 2007; 89: 780-5.

2. Kim JH, Cho MR, Kim SO, Kim JE, Lee DK, Roh WS. A comparison of femoral/sciatic nerve block with lateral femoral cutaneous nerve block and combined spinal epidural anesthesia for total knee replacement arthroplasty. Korean J Anesthesiol 2012: 62: 448-53.

3. Lang SA, Yip RW, Chang PC, Gerard MA. The femoral 3-in-1 block revisited. J Clin Anesth 1993; 5: 292-6.

4. Allen HW, Liu SS, Ware PD, Nairn CS, Owens BD. Peripheral nerve blocks improve analgesia after total knee replacement surgery. Anesth Analg 1998; 87: 93-7.

5. Macalou D, Trueck S, Meuret P, Heck M, Vial F, Ouologuem S, et al. Postoperative analgesia after total knee replacement: the effect of an obturator nerve block added to the femoral 3-in-1 nerve block. Anesth Analg 2004; 99: 251-4.

6. Bouaziz H, Vial F, Jochum D, Macalou D, Heck M, Meuret P, et al. An evaluation of the cutaneous distribution after obturator nerve block. Anesth Analg 2002; 94: 445-9.

7. Klein SM, Nielsen KC, Greengrass RA, Warner DS, Martin A, Steele SM. Ambulatory discharge after long-acting peripheral nerve blockade: 2382 blocks with ropivacaine. Anesth Analg 2002; 94: 65 70 .

8. Atkinson HD, Hamid I, Gupte CM, Russell RC, Handy JM. Postoperative fall after the use of the 3-in-1 femoral nerve block for knee surgery: a report of four cases. J Orthop Surg (Hong Kong) 2008; 16 : 381-4.

9. Williams BA, Kentor ML, Bottegal MT. The incidence of falls at home in patients with perineural femoral catheters: a retrospective summary of a randomized clinical trial. Anesth Analg 2007; 104: 1002. 